PHYSICAL REVIEW E 74, 030401(R) (2006)

\title{
Optical microscopy measurement of pair correlation functions
}

\author{
Angeles Ramírez-Saito, ${ }^{1}$ Clemens Bechinger, ${ }^{2}$ and José Luis Arauz-Lara ${ }^{1,3}$ \\ ${ }^{1}$ Instituto de Física "Manuel Sandoval Vallarta," Universidad Autónoma de San Luis Potosí, Alvaro Obregón 64, \\ 78000 San Luis Potosí, San Luis Potosí, Mexico \\ ${ }^{2}$ Physikalisches Institut, Pfaffenwaldring 57, 70550 Stuttgart, Germany \\ ${ }^{3}$ Departamento de Física, CINVESTAV, Avenida IPN 2508, Colonia Zacatenco, 07360 Mexico Distrito Federale, Mexico
}

(Received 17 March 2006; published 1 September 2006)

\begin{abstract}
We studied the pair correlation function $g(r)$ of silica particles with a fluorescent core and a nonfluorescent shell which were confined between two glass plates by optical video microscopy. To investigate the possible role of optical artifacts due to overlapping particle images, we compared experiments, where, first, the whole particle (white image) and then, only the fluorescent core (fluorescent image) was used for determining particle positions. While under white-image conditions the observed $g(r)$ exhibits a main peak at about 1.2 times the particle's diameter; under fluorescent image conditions the obtained $g(r)$ resembles a short-ranged repulsive system where the main peak is close to contact. This discrepancy points towards artifacts of video microscopy, leading to erroneous $g(r)$ and in turn to erroneous effective-pair potentials.
\end{abstract}

DOI: 10.1103/PhysRevE.74.030401

PACS number(s): 82.70.Dd, 05.40.-a

Optical microscopy methods are currently used extensively in colloidal science to determine various physical properties of colloidal systems under different conditions [1-6]. Of particular interest has been the study of quasi-twodimensional (Q2D) systems, where a colloidal suspension is confined between two parallel plates separated a distance only slightly larger than the particles' size [7-9]. Under such conditions, the particles form a single layer between the plates and one can use optical methods to image all the particles in the field of view at the same time. In this way one can directly sample the equilibrium configurations of the system which allows us to calculate several thermodynamic properties Thus, one of the key steps in those experiments is the accurate determination of the particles' positions from which various quantities of interest are determined. It has been recently demonstrated that at small particle distances under bright field illumination conditions, optical artifacts due to overlapping particle images can occur leading to an erroneous determination of the particles' positions and thus, to a deviation $\Delta r(r)$ of the actual interparticle distance [10]. Although such deviations are close to the experimental accuracy of the optical video microscopy, they can lead to considerable artifacts in other quantities, e.g., the effective-pair potential.

It has been suggested that particle image overlapping can be avoided by using core-shell particles with a fluorescent core and a nonfluorescence shell [10]. In this work we use such particles to investigate the effect of $\Delta r(r)$ on physical quantities, such as the static pair correlation function $g(r)$ in Q2D colloidal systems. This is an important issue since the pair correlation function, measured by optical microscopy, has been used in the literature to obtain a more fundamental quantity, namely, the pair interparticle potential $u(r)$ in confined geometries [7-9,11]. Here we report measurements of $g(r)$ of Q2D colloidal systems, consisting of core-shell silica spherical particles, suspended in water and confined between two glass plates. The core-shell particles' diameter is $\sigma$ $=1.4 \mu \mathrm{m}$, having a fluorescent core of diameter $400 \mathrm{~nm}$ and a nonfluorescent silica shell of a thickness of $500 \mathrm{~nm}$. The plates' separation is $1.96 \mu \mathrm{m}$. The system is observed with an optical microscope under transmission conditions with white-light illumination and independently with an epifluorescence setup. To investigate the possible role of optical artifacts due to overlapping particle images, we compared experiments where first the whole particle (white image) and then only the fluorescent core (fluorescent image) was used for determining the particle positions. As we show below, in the white-image mode, the particle images overlap when they are closer than one particle diameter, producing small distortions in the region between the particles. This, in turn, causes an incorrect determination of the particle positions and, therefore, incorrect interparticle distances [10]. On the other hand, in the fluorescent image mode the cores also appear larger than their actual size, but they are sufficiently far apart to avoid image overlapping. Pair correlation functions are determined by digitizing and analyzing a large number of particles' configurations under both conditions. This allows us to investigate the influence of the abovementioned artifacts on the resulting $g(r)$. White-imaging conditions lead to a peak in $g(r)$ at about $1.2 \sigma$, which suggests an unusual long-ranged attractive part in the pair potential. In contrast, this peak is shifted close to $\sigma$ under fluorescent imaging conditions, which is expected for shortranged repulsive particle interactions. This discrepancy points towards artifacts of video microscopy produced by overlapping particle images.

The preparation of samples, image analysis, and data processing are performed following standard procedures [12]. Core-shell particles are mixed with a small amount of polystyrene particles of diameter $h=1.96 \mu \mathrm{m}$. Prior to mixing, both species of particles are extensively dialyzed against nanopure water to reduce the ionic strength and to eliminate surfactants added by the manufacturer of the polystyrene particles. A small volume of the mixture $(\approx 1 \mu \mathrm{l})$ is confined between two clean glass plates (a slide and a cover slip), which are uniformly pressed one against the other until the separation between the plates is $h$. The larger polystyrene particles, scattered across the sample, are arrested between 
the plates and serve as spacers with an average distance in the order of $100 \mu \mathrm{m}$. The system is then sealed with epoxy resin, and the mobile core-shell particles are allowed to equilibrate in this confined geometry at room temperature $\left(25.0 \pm 0.1{ }^{\circ} \mathrm{C}\right)$. Under these conditions of confinement, the motion of the core-shell particles perpendicular to the walls is less than one particle's diameter and they form a Q2D colloidal suspension. The particles and the glass plates are negatively charged and they repel each other. Nevertheless, we added SDS (sodium dodecyl sulfate, enough to reach 1 $\mathrm{CMC}$ ) into the solution to prevent particles sticking to the walls. Although we do not add any electrolyte to the solution, it has a high ionic strength due to the ionic species dissociated from the glass walls [1]. Thus, the electrostatic repulsion is screened and the particles can approach each other near to contact. The sample is placed on the stage of an optical microscope $(100 \times$ oil immersion objective with 1.35 of numerical aperture) and observed from a top view (perpendicular to the walls plane). Images of the system with a resolution of $640 \times 480$ pixels are digitized and analyzed. Particles' $x$ and $y$ coordinates in each image are determined using the method devised by Crocker and Grier [13]. That method allows us to locate the centroid of each sphere within a precision of $1 / 5$ pixel $(\sim 0.02 \sigma)$. The pair correlation function $g(r)$, with $r$ being the measured in-plane interparticle distance, is obtained from the particles' positions in each frame and averaged to reduce the statistical noise. In order to ensure statistical independence of the particles' configurations and smooth $g(r)$ curves, runs of $10^{4}-10^{5}$ images are taken at intervals of $1 / 30 \mathrm{~s}$. The total time of each run is 100-1000 times the time decay constant of the dynamic correlation function $G(r, t)$, which is in the order of a few seconds [14]. The fluorescent cores are highly sensitive to light and they are photobleached quite easily. Thus, in the fluorescent image mode, a given site of the system can be observed only for a few minutes while the cores are still fluorescing. Then, the final results reported here are the average of measurements carried out at various sites of the system with similar particle concentration.

Let us now discuss our results. Figure 1 shows images of isolated core-shell particles taken in the white-image mode (a) and in the fluorescent image mode (b). In both cases images are radially symmetric, as shown by the corresponding light-intensity profiles (c) and (d), respectively. Although small distortions in single-particle images are sometimes observed, they appear randomly in any direction, i.e., they are due to statistical noise and have no net effect on singleparticle properties. As one can see in Fig. 1(c), the light profile in the white-image mode follows the shape of the Airy pattern. One can observe a main peak at the center followed by oscillations of decaying amplitude. On the other hand, in the fluorescent image mode, although objects appear larger than their actual size (see scale bar in the figure) there is a sharper cutoff between the bright fluorescing object and the dark background. Here one can see only a main peak whose intensity decays monotonically, i.e., without oscillations, towards the background.

Figure 2 shows images of a pair of core-shell particles separated a distance between surfaces less than one particle's
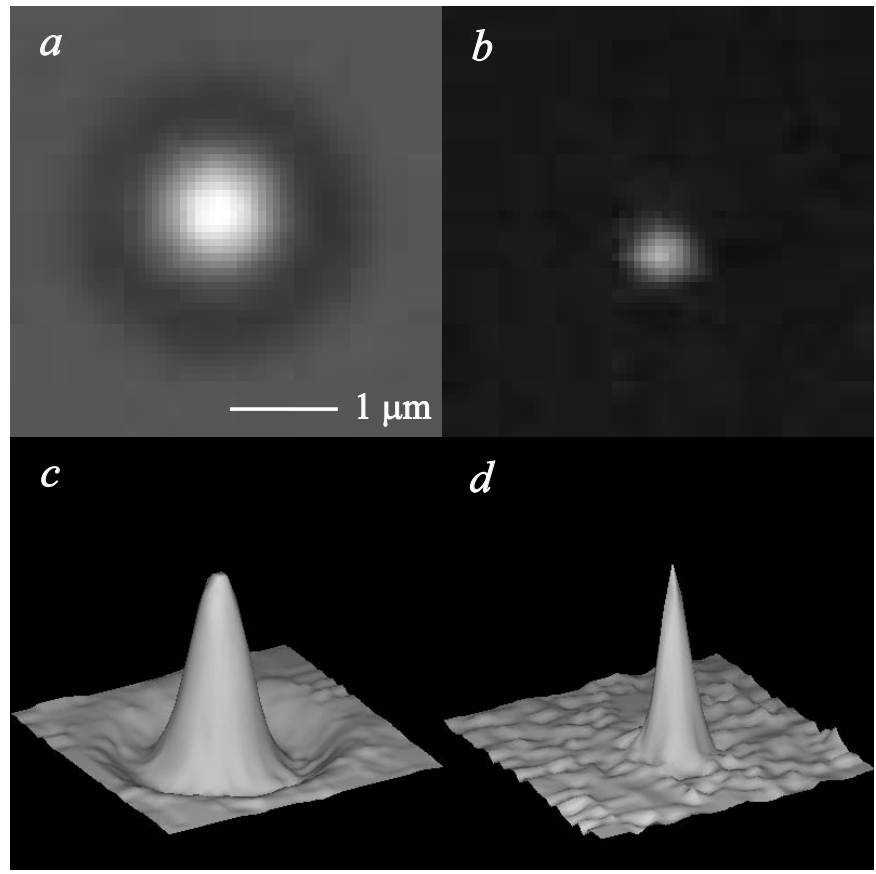

FIG. 1. Optical microscope images of an isolated core-shell particle observed in the white-image (a) and fluorescent image (b) mode. Images (c) and (d) are the light-intensity profiles of (a) and (b), respectively.

diameter taken in the white-image mode (a) and then taken in the fluorescent-image mode (b). Since particles diffuse, they are not at exactly the same distance in both images. The light-intensity profiles are plotted in (c) and (d), respectively. As one can see in 2(a), in the white-image mode there is an

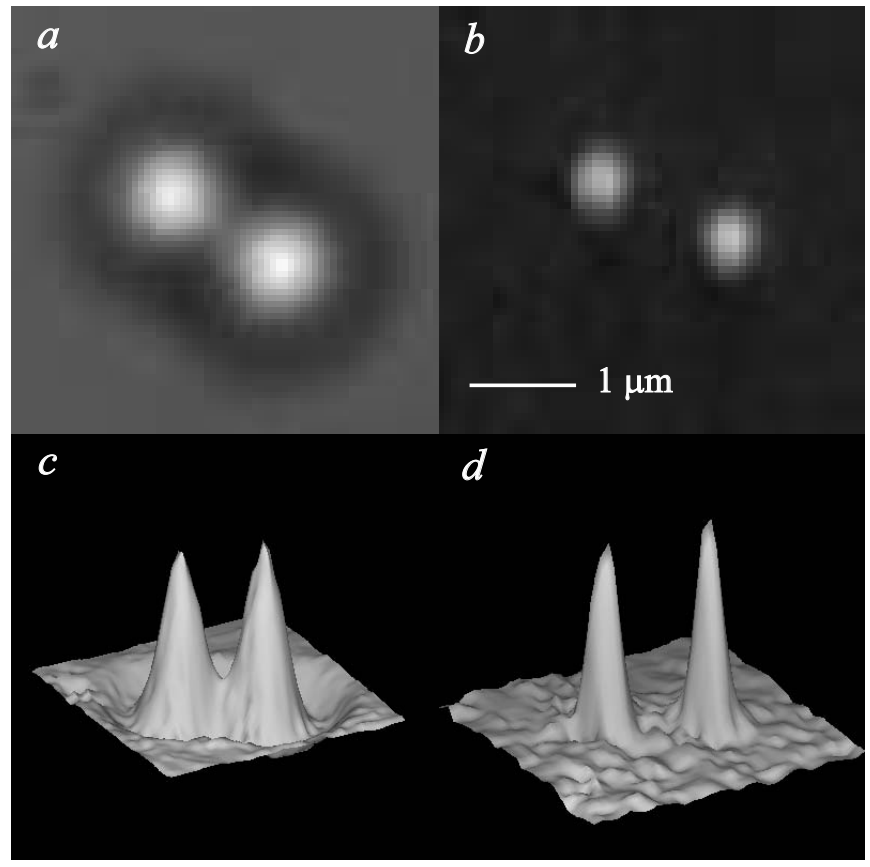

FIG. 2. Optical microscope images of a pair of core-shell particles observed in the white-image (a) and fluorescent image (b) mode. Images (c) and (d) are the light-intensity profiles of (a) and (b), respectively. 


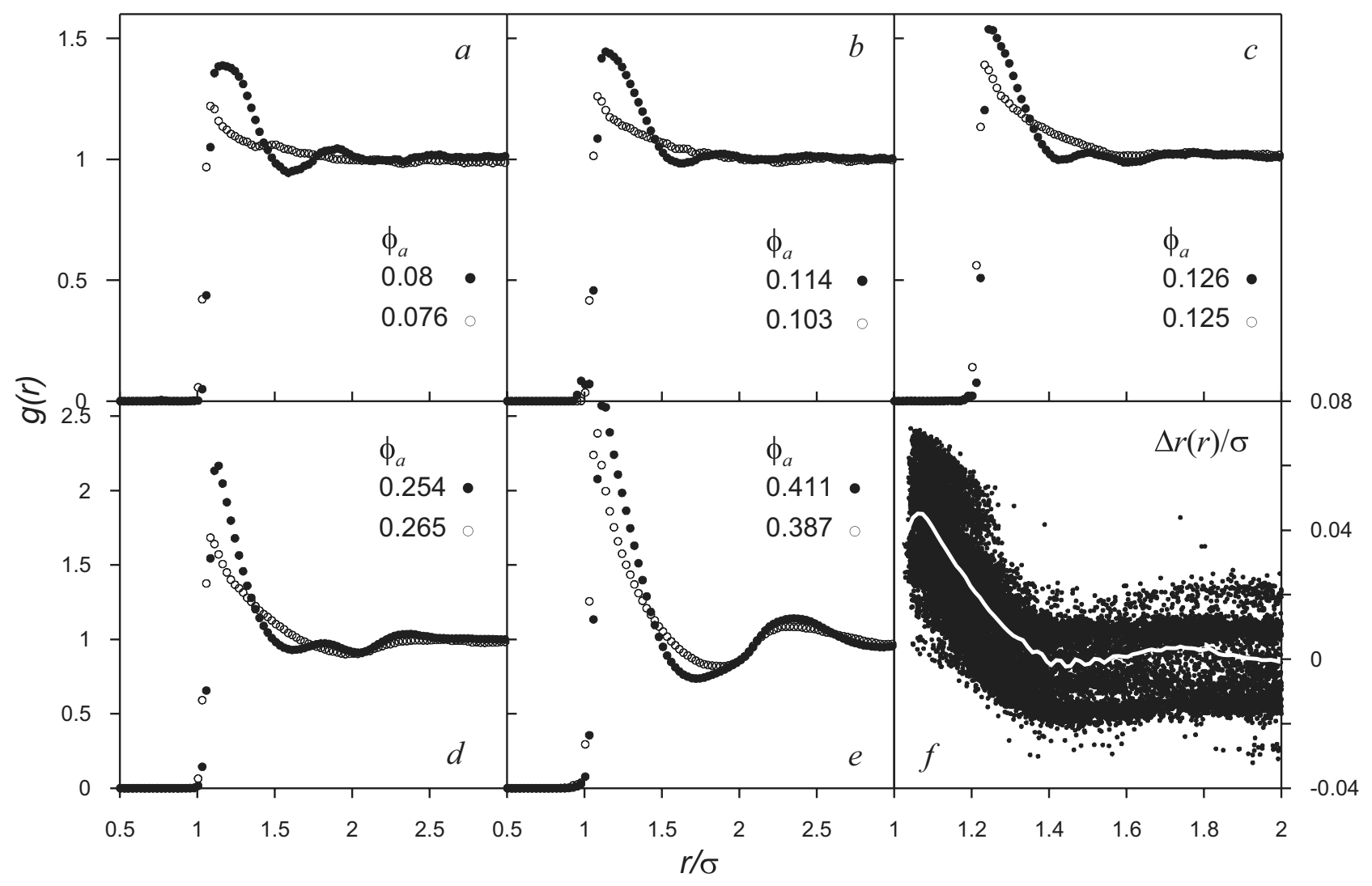

FIG. 3. Pair correlation function of Q2D suspensions of core-shell silica particles for different values of the particle area fraction $\phi_{a}$, measured by optical microscopy in the white-image mode (solid circles) and in the fluorescent image mode (open symbols). (f) shows the interparticle distance deviation $\Delta r(r)$. Symbols represent independent measurements performed in a thin cell sample of $1.5 \mu \mathrm{m}$ silica particles suspended in water; the solid line is the average.

overlap of the single-particle light-intensity profiles, which are of longer range than the particle's physical diameter [see Fig. 1(c)], producing a small distortion of the image along the line joining the particles' centroids. Such distortion is shown in more detail in the light-intensity profile in Fig. 2(c). As one can see here, the intensity profiles of individual particles are no longer radially symmetric, the peaks are somewhat distorted (the tip is shifted), and there is an intensity overlap in between the particles. The quantitative effect of such distortion is a shift in the position of the intensity weighted centroid, leading to a deviation of the interparticle distance, as determined by a numerical image analysis. Such deviation depends on the interparticle distance and its magnitude is in the order of the experimental accuracy. On the other hand, in the fluorescent image mode one sees only the images of the fluorescent cores, which are still radially symmetric as shown in Figs. 2(b) and 2(d). Since the nonfluorescent silica shell covering the fluorescent cores is thicker than the core's diameter, the cores are always sufficiently far apart to avoid image overlapping, even when the particles are at contact. Therefore, in this mode, one can expect image analysis to provide the true particle position and accurate interparticle distances.

Although minute, the deviation of the interparticle distances described above can produce a dramatic cumulative effect on physical quantities, such as the static pair correlation function $g(r)$ as it is shown in Fig. 3. Here we present the pair correlation function of Q2D colloidal suspensions of core-shell particles, measured by optical microscopy in the white-image mode and in the fluorescent image mode for various particle area fractions $\phi_{a}$. Closed circles in Figs. 3(a)-3(e) represent the results from the white-image mode. As one can see here, $g(r)$ follows a similar qualitative behavior within the range of particle concentrations studied, namely, it raises smoothly at interparticle distances $r>\sigma$, reaching a maximum, and at larger distances it oscillates around its asymptotic value of 1 . The main peak is located at a distance $r \sim 1.2 \sigma$, depending only slightly on particle concentration. Let us note that similar qualitative behavior of the pair correlation function has been reported in Q2D aqueous suspensions of nonfluorescent polystyrene and silica spheres, as well as in suspensions of fluorescent polystyrene spheres, measured by optical microscopy in the white-image mode and in the fluorescent image mode, respectively [7-9]. Those experiments suggested an attractive component in the pair interaction between negatively charged colloidal particles in confinement. On the other hand, open circles in Figs. 3(a)-3(e) represent the pair correlation function of the coreshell particles measured in the fluorescent image mode. As one can see in Fig. 3, both measurements of $g(r)$ differ quite dramatically from each other near contact $(\sigma<r<2 \sigma)$. In the fluorescent image mode, the measured pair correlation function does not exhibit the peak at $r \sim 1.2 \sigma$, but instead there appears a peak near contact and $g(r)$ resembles the pair 
correlation function of hard disks. Figure 3(f) shows the interparticle distance deviation $\Delta r(r)=r-\left[\left(x-x_{r e f}\right)^{2}+(y\right.$ $\left.\left.-y_{\text {ref }}\right)^{2}\right]^{1 / 2}$ measured in the way discussed in Ref. [10], using $1.5 \mu \mathrm{m}$ silica particles. Here $r$ is the measured interparticle distance between a mobile particle of coordinates $(x, y)$ and a fixed particle whose true position $\left(x_{r e f}, y_{r e f}\right)$ is determined when the mobile particle is far away. As discussed in [10], the true distance between two isolated particles is given by $r_{T}=r-2 \Delta r(r)$. In Fig. 3(f) symbols represent independent measurements of $\Delta r(r)$ for interparticle distances between 1 and 2 particle's diameter and the continuous line is the average. Since the pair correlation function $g(r)$ is determined by counting the number of particles at a distance $r$ of a given particle, and normalizing the histogram with the histogram of an ideal gas, it depends sensitively on the accurate measurement of interparticle distances. The fact that the deviation $\Delta r(r)$ is a non linear function of $r$ produces an artificial accumulation in the histogram at $r \sim 1.2 \sigma$, at the expenses of its value at other interparticle distances. The net effect is then the appearance of an artificial peak of $g(r)$ as shown in Fig. 3.

Summary. We report here the observation of small distortions in the images of pairs of colloidal particles obtained by transmitted white-light optical microscopy when their surfaces are closer than one particle's diameter. Those distortions, due to the superposition of particles' images, shift the apparent particle positions, producing deviations in the interparticle distances when they are close to contact. Although small, such deviations can add up its effect and lead to the appearance of fictitious features in static structural quantities such as the pair correlation function. Thus, our work points out those technical artifacts and provides a way to avoid them.

We acknowledge Alfons van Blaaderen (Utrecht) for providing the core-shell particles used in this work and Jörg Baumgartl for helpful discussions. This work is financially supported by Consejo Nacional de Ciencia y Tecnología, Mexico, Grant No. SEP-2004-C01-46121.
[1] C. A. Murray and D. G. Grier, Annu. Rev. Phys. Chem. 47, 421 (1996).

[2] D. T. Wasan and A. D. Nikolov, Nature (London) 423, 156 (2003).

[3] K. Zahn, A. Wille, G. Maret, S. Sengupta, and P. Nielaba, Phys. Rev. Lett. 90, 155506 (2003).

[4] P. N. Pusey, Science 309, 1198 (2005).

[5] K. Kang, J. Gapinski, M. P. Lettinga, J. Buitenhuis, G. Meier, M. Ratajczyk, J. K. G. Dhont, and A. Patkowski, J. Chem. Phys. 122, 044905 (2005).

[6] C.-Y. Chou, B. C. Eng, and M. Robert, J. Chem. Phys. 124, 044902 (2006).

[7] G. M. Kepler and S. Fraden, Phys. Rev. Lett. 73, 356 (1994).

[8] M. D. Carbajal-Tinoco, F. Castro-Román, and J. L. Arauz-
Lara, Phys. Rev. E 53, 3745 (1996).

[9] S. H. Behrens and D. G. Grier, Phys. Rev. E 64, 050401(R) (2001).

[10] J. Baumgartl and C. Bechinger, Europhys. Lett. 71, 487 (2005); J. Baumgartl, J. L. Arauz-Lara, and C. Bechinger, Soft Matter 2, 631 (2006)

[11] B. Cui, B. Lin, S. Sharma, and S. A. Rice, J. Chem. Phys. 116, 3119 (2002).

[12] A. Ramírez-Saito, M. Chávez-Páez, J. Santana-Solano, and J. L. Arauz-Lara, Phys. Rev. E 67, 050403(R) (2003).

[13] J. C. Crocker and D. G. Grier, J. Colloid Interface Sci. 179, 298 (1996).

[14] J. Santana-Solano and J. L. Arauz-Lara, Phys. Rev. Lett. 87, 038302 (2001). 